\title{
Thiazolidinedione increases serum soluble receptor for advanced glycation end-products in type 2 diabetes
}

\author{
K. C. B. Tan • W. S. Chow • A. W. K. Tso • A. Xu • \\ H. F. Tse • R. L. C. Hoo • D. J. Betteridge • K. S. L. Lam
}

Received: 23 February 2007 / Accepted: 6 June 2007 / Published online: 18 July 2007

(C) Springer-Verlag 2007

\begin{abstract}
Aims/hypothesis Interfering with the activation of receptor for AGE (RAGE) by using a soluble form of the AGE receptor (sRAGE) prevents or ameliorates the vascular complications of diabetes in experimental studies. Relatively little is known about factors that influence endogenous circulating sRAGE in humans. We investigated the impact of improving glycaemic control on serum total sRAGE and endogenous secretory RAGE (esRAGE), a splice variant of sRAGE, and compared the effect of rosiglitazone with that of sulfonylurea.

Methods A randomised, open-label, parallel group study was performed with 64 participants randomised to receive add-on therapy with either rosiglitazone or sulfonylurea. Serum total sRAGE and esRAGE and metabolic parameters were measured before and after 6 months of treatment.

Results At 6 months, both rosiglitazone and sulfonylurea resulted in a significant reduction in $\mathrm{HbA}_{1 \mathrm{c}}$, fasting glucose
\end{abstract}

K. C. B. Tan $(\bowtie) \cdot$ W. S. Chow $\cdot$ A. W. K. Tso $\cdot$ A. Xu $\cdot$

H. F. Tse $\cdot$ R. L. C. Hoo $\cdot$ K. S. L. Lam

Department of Medicine, University of Hong Kong,

Queen Mary Hospital,

Pokfulam Road,

Hong Kong SAR, People's Republic of China

e-mail: kcbtan@hkucc.hku.hk

K. C. B. Tan $\cdot$ A. Xu $\cdot$ H. F. Tse $\cdot$ K. S. L. Lam

Research Centre of Heart, Brain, Hormone and Healthy Aging,

University of Hong Kong,

Hong Kong SAR, People's Republic of China

D. J. Betteridge

Department of Medicine,

Royal Free and University College London Medical School,

London, UK and AGE. However, significant increases in total sRAGE and esRAGE were only seen in the rosiglitazone group. As a result, serum esRAGE was higher in the rosiglitazone group than in the sulfonylurea group at 6 months $(p<0.01)$, whereas the differences in sRAGE between the two groups did not reach statistical significance. Stepwise linear regression analysis showed that treatment modality made a greater contribution than the changes in $\mathrm{HbA}_{1 \mathrm{c}}$ to the subsequent changes in esRAGE levels at 6 months.

Conclusions/interpretation Treating type 2 diabetic patients with thiazolidinedione can increase circulating levels of esRAGE and sRAGE. Whether modulation of circulating sRAGE has a beneficial effect on diabetic complications will have to be evaluated in long-term prospective studies.

International Standard Randomised Controlled Trial Number ISRCTN05215453.

Keywords Advanced glycation end-products · RAGE . Soluble receptor for advanced glycation end-products . Thiazolidinedione · Type 2 diabetes mellitus

$\begin{array}{ll}\text { Abbreviations } \\ \text { CML } & N^{\varepsilon} \text {-(carboxymethyl)lysine } \\ \text { esRAGE } & \begin{array}{l}\text { endogenous secretory receptor for advanced } \\ \text { glycation end-products }\end{array} \\ \text { HOMA- } & \begin{array}{l}\text { homeostasis model assessment index of insulin } \\ \text { resistance }\end{array} \\ \text { IR } & \text { malondialdehyde } \\ \text { MDA } & \text { peroxisome proliferator-activated receptor } \gamma \\ \text { PPAR } \gamma & \begin{array}{l}\text { receptor for advanced glycation end-products } \\ \text { RAGE } \\ \text { sRAGE }\end{array} \\ & \begin{array}{l}\text { soluble receptor for advanced glycation end- } \\ \text { products }\end{array}\end{array}$




\section{Introduction}

Hyperglycaemia leads to the increased formation and accumulation of AGE, the binding of which to their cellular receptors plays an important role in the pathogenesis of diabetic vascular complications $[1,2]$. A variety of AGE receptors or AGE-binding proteins have been described. Among them, the receptor for AGE (RAGE) is the best characterised molecule [3]. RAGE belongs to the immunoglobulin superfamily of cell-surface molecules and recent studies have identified novel splice variants of the human RAGE receptor [4-6]. Three major RAGE (also known as $A G E R)$ mRNA variants have been identified. One of these encodes the full-length RAGE, which has full signalling and AGE-binding potential. Another encodes N-truncated RAGE, a membrane-bound isoform containing no AGEbinding domain. The third encodes $\mathrm{C}$-truncated soluble RAGE (sRAGE), which has AGE-binding properties in the absence of a signalling cascade.

The engagement of the full-length RAGE by AGE activates key cell signalling pathways such as mitogenactivated protein kinases and nuclear factor- $\mathrm{kB}$, resulting in a perturbation of a variety of homeostatic functions of the vasculature and induction of cellular oxidant stress $[1,7]$. Interfering with the activation of RAGE by using recombinant sRAGE or function-blocking antibodies suppresses vascular hyperpermeability, atherosclerotic lesion development and enhances wound healing in diabetic rodents [810]. A splice variant that directed the synthesis of sRAGE proteins in cultured human microvascular endothelial cells, named endogenous secretory RAGE (esRAGE) by the investigators, was also able to capture AGE ligands and neutralise the action of AGE on endothelial cells [4]. Taken together, these data suggest that sRAGE may have a protective function against the development of diabetic vascular complications by acting as a decoy for RAGE ligands; it thus may have cytoprotective properties against AGE actions.

There are only limited data on sRAGE in human studies. Cross-sectional studies in human diabetic patients have suggested that serum sRAGE levels were increased in type 1 and type 2 diabetic patients with complications [11, 12]. On the other hand, studies that measured esRAGE found lower esRAGE levels in type 1 and type 2 diabetic patients $[13,14]$. Since there are a number of distinct splice variants of sRAGE [4-6], it has been suggested that esRAGE might constitute only part, but not all of the human sRAGE in plasma. Relatively little is known about factors that influence endogenous circulating sRAGE levels; however, an association with glycaemic control has recently been reported [15]. Diverse variants of RAGE may be found in many tissues and cell types and it has been suggested that the pre-mRNA of $R A G E$ must be subjected to regulated alternative splicing activated by extracellular cues of yet unknown cellular signalling pathways [5]. Recent studies have suggested that activation of peroxisome proliferatoractivated receptor $\gamma$ (PPAR $\gamma)$ can modulate full-length RAGE expression in endothelial cells and vascular smooth muscle cells [16, 17], but its effect on sRAGE has not been determined. Hence, in the present study, we investigated the impact of improving glycaemic control on esRAGE and total sRAGE, and compared the effect of rosiglitazone with that of sulfonylurea.

\section{Methods}

Participants The effect of rosiglitazone on esRAGE and total sRAGE was compared with that of sulfonylurea in a randomised, open-label, parallel group study. Chinese type 2 diabetic patients who had been treated with diet alone, metformin and/or sulfonylurea $<$ half-maximum dose for at least 6 months with suboptimal glycaemic control $\left(\mathrm{HbA}_{1 \mathrm{c}} \geq\right.$ $7.5 \%)$ were randomised to receive add-on therapy with either rosiglitazone $4 \mathrm{mg}$ or glibenclamide $5 \mathrm{mg}$ (or gliclazide $80 \mathrm{mg}$ ) daily, while keeping the doses of their usual glucose-lowering agents constant. After 4 weeks, the doses of the add-on therapy were doubled in participants with fasting blood glucose level $>8.0 \mathrm{mmol} / \mathrm{l}$ and without symptomatic or asymptomatic hypoglycaemia, defined as blood glucose level $<3.0 \mathrm{mmol} / \mathrm{l}$. The dosages of all glucose-lowering agents were then kept constant for another 20 weeks. Power calculation showed that a sample size of 32 participants in each group was required to detect a difference of $20 \%$ in esRAGE level between the two groups, with $80 \%$ power at a $5 \%$ alpha level. Individuals with $\mathrm{HbA}_{1 \mathrm{c}}>10.5 \%$, blood pressure $>160 / 90 \mathrm{mmHg}$ or impaired renal or liver function were excluded. The dosages of any lipid-lowering and anti-hypertensive agents were kept unchanged throughout the study period. Fasting blood samples were taken at baseline and at the end of the study (24 weeks). The study protocol was approved by the Institutional Review Board of the University of Hong Kong, and written informed consent was obtained from all participants.

Laboratory procedures Laboratory personnel were blinded to the treatment status of the participants. Samples were assayed in duplicate and in random order. Serum sRAGE levels were determined using ELISA (Quantikine; R\&D systems, Minneapolis, MN, USA) according to the manufacturer's protocol. This assay measures the serum total sRAGE level. Briefly, a monoclonal antibody raised against the N-terminal extracellular domain of human RAGE consisting of amino acids Gln24 through to Ala344 was used to capture sRAGE from serum. Captured sRAGE was 
detected with a polyclonal antihuman sRAGE antibody raised against the same immunogen. After washing, plates were incubated with streptavidin-horseradish peroxidase, developed with appropriate substrate, and then the optical density at $450 \mathrm{~nm}\left(\mathrm{OD}_{450}\right)$ was determined using an ELISA plate reader. The intra- and inter-assay coefficients of variation were 1.9 and $5.5 \%$, respectively. Serum esRAGE levels were determined using a different ELISA (B-Bridge International, Mountain View, CA, USA). The capture antibody in the ELISA assay for esRAGE uses the monoclonal antibody 278-13G4 and the detection antibody is a rabbit esRAGE-specific polyclonal antibody raised against the unique C-terminal 16-amino-acid peptide (amino acids 332-347) of esRAGE. The intra- and inter-assay coefficients of variation were 3.7 and $5.4 \%$, respectively.

$\mathrm{HbA}_{1 \mathrm{c}}$ was measured in whole blood using ion-exchange high-performance liquid chromatography with a haemoglobin testing system (Variant; Bio-Rad Laboratories, Hercules, CA, USA). Serum AGE were measured by competitive ELISA using a well-characterised polyclonal rabbit antiserum raised against AGE-RNase as previously described [18]. Plasma total cholesterol and triacylglycerol were determined enzymatically on an analyser (Hitachi 912; Roche Diagnostics, Mannheim, Germany). HDL-cholesterol was measured using a homogenous method with polyethylene glycol-modified enzymes and alpha-cyclodextrin. LDLcholesterol was calculated by the Friedewald equation. Serum insulin was assayed by microparticle enzyme immunoassay (Abbott Laboratories, Tokyo, Japan). Homeostasis model assessment index of insulin resistance (HOMA-IR) was calculated to estimate insulin resistance (for HOMA calculator see www.dtu.ox.ac.uk, last accessed in June 2007). Serum adiponectin level was determined with an in-house ELISA assay [19]. Plasma high-sensitivity C-reactive protein was measured with a particle-enhanced immunoturbidimetric assay (Roche Diagnostics). Malondialdehyde (MDA), a marker of lipid peroxidation and an equivalent of thiobarbituric acid reactive substances in serum, was measured using an assay kit (Oxi-Tek TBARS; Alexis Biochemicals, Lausen, Switzerland).

Statistical analysis Results are expressed as means \pm SD or medians and interquartile range if data were not normally distributed. All data were tested for normality using the Kolmogorov-Smirnov test. Comparison of variables between two groups was performed using two sample $t$ test; comparisons of these variables before and after treatment were analysed by paired $t$ test. Skewed data were logarithmically transformed before analysis if necessary. Spearman correlation analysis was used to test the relationship between variables. Multiple linear regression was used to analyse the relationship between changes in glycaemic control and the effect of different treatment modalities on serum esRAGE and total sRAGE. In variables where the distribution of the magnitude of change after treatment was not normally distributed, log-change ratio was used in the regression analysis.

\section{Results}

Baseline clinical characteristics, metabolic profiles and medications were similar between the two groups except for the higher proportion of smokers and higher triacylglycerol levels in the rosiglitazone group (Table 1). Serum esRAGE level and total sRAGE level were comparable in the two groups at baseline and there were no significant differences between individuals on sulfonylurea monotherapy compared with those on a combination of sulfonylurea and metformin (Table 2). Serum esRAGE level was about one-third that of total sRAGE and they were closely correlated ( $r=0.83$ and $r=0.84$ in the rosiglitazone and sulfonylurea group, respectively). At the end of the study period, both rosiglitazone and sulfonylurea resulted in a significant reduction in $\mathrm{HbA}_{1 \mathrm{c}}$, fasting glucose, AGE and MDA (Table 2). However, a significant increase in total sRAGE and esRAGE was seen only in the rosiglitazone group (Fig. 1a,b). As a result, serum esRAGE was significantly higher in the rosiglitazone group than in the sulfonylurea group at 24 weeks, a difference that remained significant after adjusting for smoking status, sex and triacylglycerol levels. The observed effects were not dependent on baseline therapy (sulfonylurea monotherapy or sulfonylurea and metformin combination therapy). The dose of rosiglitazone was increased to $8 \mathrm{mg}$ per day in $50 \%$ of the participants after week 4. At 6 months, these individuals had higher levels of sRAGE $(955.4 \pm 343.0$ $\mathrm{pg} / \mathrm{ml}$ vs $702.0 \pm 315.6, p=0.04)$ and esRAGE $(345.1 \pm$ $156.5 \mathrm{pg} / \mathrm{ml}$ vs $250.7 \pm 121.2, p=0.06)$ than those who remained on the lower dose of $4 \mathrm{mg}$ daily. In the sulfonylurea group, $50 \%$ of the participants also had a dosage increase at week 4 , but there were no significant differences in sRAGE and esRAGE levels at 6 months between the two groups on different dosages. Plasma adiponectin increased in both the rosiglitazone and sulfonylurea groups; in the former there was a reduction in fasting insulin level, too.

Table 3 shows the magnitude of change of esRAGE, total sRAGE and metabolic parameters. The reduction in fasting glucose was statistically significantly greater in the rosiglitazone group, but the changes in $\mathrm{HbA}_{1 \mathrm{c}}$ were similar. There were no significant differences in the reductions in AGE and MDA between the two groups, whereas the increase in plasma adiponectin was much more pronounced in the rosiglitazone group. Correlation analysis was performed to determine whether the changes in total 
Table 1 Clinical characteristics of the two groups of diabetic patients

\begin{tabular}{|c|c|c|c|c|}
\hline & \multicolumn{2}{|l|}{ Baseline } & \multicolumn{2}{|l|}{6 months } \\
\hline & Rosiglitazone & Sulfonylurea & Rosiglitazone & Sulfonylurea \\
\hline Men/women & $21 / 11$ & $15 / 17$ & - & - \\
\hline Age (years) & $55.4 \pm 9.8$ & $57.1 \pm 10.0$ & - & - \\
\hline Duration of diabetes (years) & $10.1 \pm 5.0$ & $9.0 \pm 3.8$ & - & - \\
\hline Smoker $(\%)$ & $22 *$ & 9 & - & - \\
\hline Diet/sulfonylurea/combination $(\%)^{\mathrm{a}}$ & $0 / 25 / 75$ & $3 / 22 / 75$ & - & - \\
\hline ACEI or ARB $(\%)$ & 31 & 38 & - & - \\
\hline Statin or fibrate $(\%)$ & 22 & 25 & - & - \\
\hline BMI $\left(\mathrm{kg} / \mathrm{m}^{2}\right)$ & $25.39 \pm 3.57$ & $25.85 \pm 3.93$ & $25.95 \pm 3.71$ & $25.28 \pm 4.17$ \\
\hline Waist circumference $(\mathrm{cm})$ & $86.9 \pm 10.1$ & $88.3 \pm 11.9$ & $87.4 \pm 10.5$ & $86.8 \pm 12.3$ \\
\hline Systolic BP (mmHg) & $129.2 \pm 14.7$ & $125.3 \pm 14.9$ & $124.5 \pm 12.5$ & $121.6 \pm 13.6$ \\
\hline Diastolic BP (mmHg) & $79.1 \pm 7.1$ & $78.8 \pm 7.9$ & $75.5 \pm 5.9$ & $74.3 \pm 8.3$ \\
\hline $\mathrm{TC}(\mathrm{mmol} / \mathrm{l})$ & $5.13 \pm 1.07$ & $4.76 \pm 0.78$ & $5.22 \pm 1.09$ & $4.59 \pm 0.83$ \\
\hline TG (mmol/l) & $1.60(1.10-2.25)^{*}$ & $1.10(0.90-1.48)$ & $1.50(1.10-2.40)^{*}$ & $1.05(0.70-1.55)$ \\
\hline LDL-cholesterol (mmol/l) & $3.09 \pm 0.93$ & $2.82 \pm 0.71$ & $3.17 \pm 0.93$ & $2.61 \pm 0.62$ \\
\hline HDL-cholesterol (mmol/1) & $1.21 \pm 0.31$ & $1.36 \pm 0.37$ & $1.27 \pm 0.34$ & $1.37 \pm 0.45$ \\
\hline
\end{tabular}

Values are means \pm SD if normally distributed or medians (interquartile range) if skewed. Skewed data were logarithmically transformed before analysis.

$A C E I$ ACE inhibitor; $A R B$ angiotensin receptor blocker; $T C$ total cholesterol; $T G$ triacylglycerol

$* p<0.05$ vs sulfonylurea group

${ }^{\mathrm{a}}$ Combination: sulfonylurea and metformin

sRAGE and esRAGE were related to the changes in metabolic parameters (Table 4). The increase in esRAGE was related to the reduction in $\mathrm{HbA}_{1 \mathrm{c}}$ in the rosiglitazone group, whereas in the sulfonylurea group the changes in total sRAGE and esRAGE were related to the reduction in fasting glucose and MDA.

To determine the major determinants of the changes in esRAGE, stepwise linear regression analysis was performed. Age, sex, BMI, smoking status, changes in $\mathrm{HbA}_{1 \mathrm{c}}$, changes in MDA and treatment modality (rosiglitazone/ sulfonylurea) were entered into the model. Only treatment modality (standardised coefficient $\beta=0.332, p=0.005, R^{2}=$ 0.20 ) and the changes in $\mathrm{HbA}_{1 \mathrm{c}}$ (standardised coefficient $\left.\beta=-0.296, p=0.023, R^{2}=0.08\right)$ were significant independent determinants of the changes in esRAGE (total model $R^{2}=0.28$ ). An interaction between changes in $\mathrm{HbA}_{1 \mathrm{c}}$ and treatment modality was tested and no significant interaction found; the contribution of treatment modality to the

Table 2 Indices of glycaemic control, serum total sRAGE, serum esRAGE and metabolic parameters before and after treatment

\begin{tabular}{|c|c|c|c|c|}
\hline & \multicolumn{2}{|l|}{ Baseline } & \multicolumn{2}{|l|}{6 months } \\
\hline & Rosiglitazone & Sulfonylurea & Rosiglitazone & Sulfonylurea \\
\hline $\mathrm{HbA}_{1 \mathrm{c}}(\%)$ & $8.67 \pm 0.74$ & $8.50 \pm 0.61$ & $7.62 \pm 0.89^{* *}$ & $7.85 \pm 0.82 * *$ \\
\hline Fasting glucose $(\mathrm{mmol} / \mathrm{l})$ & $8.69 \pm 1.52$ & $8.62 \pm 1.93$ & $7.04 \pm 1.58^{* *}$ & $7.79 \pm 1.32 * *$ \\
\hline AGE (unit/ml) & $5.42 \pm 1.46$ & $5.44 \pm 1.36$ & $4.92 \pm 1.33 * *$ & $5.08 \pm 1.49^{*}$ \\
\hline SRAGE $(\mathrm{pg} / \mathrm{ml})$ & $708.1 \pm 240.0$ & $719.0 \pm 251.6$ & $824.6 \pm 348.2 * *$ & $702.6 \pm 260.8$ \\
\hline EsRAGE (pg/ml) & $243.8 \pm 95.4$ & $222.3 \pm 91.7$ & $296.4 \pm 145.2 * *, * * *$ & $213.9 \pm 81.2$ \\
\hline Adiponectin $(\mu \mathrm{g} / \mathrm{ml})$ & $5.95(4.16-10.65)$ & $6.51(4.16-10.50)$ & $10.62(6.27-23.49)^{* *}$ & $7.66(5.06-10.55)^{*}$ \\
\hline Fasting insulin (pmol/l) & $73.32 \pm 36.38$ & $65.56 \pm 34.54$ & $62.56 \pm 29.03 *$ & $74.05 \pm 53.80$ \\
\hline HOMA-IR & $3.60(2.29-4.66)$ & $2.91(2.01-4.90)$ & $2.45(1.77-3.68)^{* *}$ & $3.14(1.53-5.16)$ \\
\hline MDA (nmol/ml) & $6.62 \pm 2.61$ & $6.45 \pm 1.99$ & $5.68 \pm 1.81 * *$ & $5.69 \pm 1.60 * *$ \\
\hline C-reactive protein $(\mathrm{mg} / \mathrm{l})$ & $1.48(0.58-2.71)$ & $0.97(0.54-2.79)$ & $0.95(0.50-2.72)$ & $1.00(0.67-2.04)$ \\
\hline
\end{tabular}

Values are means \pm SD if normally distributed, or medians (interquartile range) if skewed. Skewed data were logarithmically transformed before analysis.

*Paired $t$ test: $p<0.05$

**Paired $t$ test: $p<0.01$ for within-group comparison vs baseline

***Two sample $t$ test: $p<0.01$ vs sulfonylurea group at 6 months 

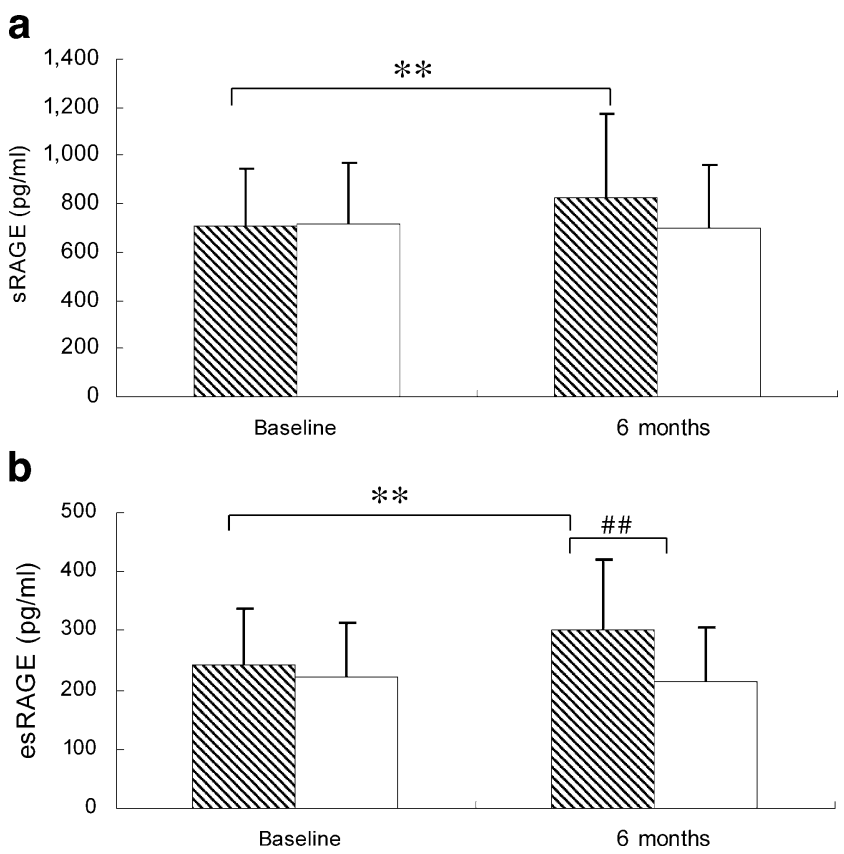

Fig. 1 Serum concentrations of total sRAGE (a) and esRAGE (b) at baseline and 6 months. Values are means \pm SD. Hatched bars, rosiglitazone; open bars, sulfonylurea. Asterisks, $p<0.01$ rosiglitazone group at 6 months vs baseline; hash symbols, $p<0.01$ rosiglitazone group at 6 months vs sulfonylurea group at 6 months

changes in esRAGE levels was greater than that of changes in $\mathrm{HbA}_{1 \mathrm{c}}$. All analyses were also repeated using the changes in fasting glucose instead of $\mathrm{HbA}_{1 \mathrm{c}}$, but the findings remained the same.

\section{Discussion}

There is substantial experimental evidence to suggest that blockade of RAGE by the soluble form of its receptor can prevent or ameliorate the vascular complications of diabetes

Table 3 The magnitude of change in the indices of glycaemic control, serum total sRAGE, serum esRAGE and metabolic parameters in the two groups of participants

\begin{tabular}{lll}
\hline & Rosiglitazone & Sulfonylurea \\
\hline HbA $_{1 \mathrm{c}}$ & $-10.6(-18.2$ to -5.7$)$ & $-8.4(-14.7$ to -0.8$)$ \\
Fasting glucose & $-17.1(-31.8 \text { to }-12.2)^{*}$ & $-8.9(-22.9$ to 1.9$)$ \\
AGE & $-5.6(-19.4$ to 2.1$)$ & $-4.2(-15.4$ to 11.2$)$ \\
SRAGE & $14.7(-1.4 \text { to } 30.2)^{*}$ & $-4.4(-12.2$ to 5.2$)$ \\
esRAGE & $7.9(0.6 \text { to } 40.0)^{*}$ & $-4.8(-13.1$ to 9.4$)$ \\
Adiponectin & $67.1(44.6 \text { to } 173.5)^{*}$ & $7.6(-7.4$ to 39.3$)$ \\
Insulin & $-6.9(-33.8$ to 14.3$)$ & $-11.1(-21.6$ to 34.4$)$ \\
HOMA-IR & $-29.5(-46.5$ to -7.1$)$ & $-16.8(-32.7$ to 31.4$)$ \\
MDA & $-5.6(-19.4$ to 2.1$)$ & $-10.7(-19.5$ to 0.5$)$ \\
\hline
\end{tabular}

Values (percentage change from baseline) are medians (interquartile range); data were analysed by Mann-Whitney $U$ test ${ }^{*} p<0.01$ vs sulfonylurea group
Table 4 Relationship of the changes in serum total sRAGE and esRAGE with changes in the various metabolic parameters

\begin{tabular}{llllll}
\hline & \multicolumn{2}{l}{ Rosiglitazone } & & \multicolumn{2}{l}{ Sulfonylurea } \\
\cline { 2 - 3 } \cline { 5 - 6 } \cline { 5 - 6 } & sRAGE & esRAGE & & sRAGE & esRAGE \\
\hline HbA $_{1 \mathrm{c}}$ & -0.19 & $-0.44^{*}$ & & -0.20 & -0.34 \\
Fasting glucose & -0.16 & -0.24 & & $-0.43^{*}$ & $-0.57^{* *}$ \\
AGE & 0.23 & 0.10 & & -0.18 & -0.30 \\
Adiponectin & 0.01 & 0.19 & & -0.08 & 0.11 \\
Insulin & -0.30 & -0.22 & & -0.18 & -0.02 \\
HOMA-IR & -0.28 & -0.30 & & -0.31 & -0.26 \\
MDA & -0.19 & -0.10 & & $-0.45^{* *}$ & $-0.37^{*}$ \\
\hline
\end{tabular}

Values are Spearman correlation coefficients

$* p<0.05$

$* * p<0.01$

$[8,10]$. However, the mechanisms by which sRAGE might be generated in vivo remain poorly understood. It has been suggested that human sRAGE production might be regulated by alternative splicing and by release from the fulllength RAGE receptor by proteinases [4-6, 20, 21]. It is not known what controls the alternative splicing of the $R A G E$ pre-mRNA and the pattern of production of the different RAGE isoforms.

This is the first study to examine the impact of improving glycaemic control on total sRAGE and esRAGE levels and compare the effects of thiazolidinedione with sulfonylurea. Despite a similar degree of improvement in glycaemic control in both groups, a significant increase in total sRAGE and esRAGE was only seen in the rosiglitazone group, with participants on the larger dose of rosiglitazone having higher levels of sRAGE and esRAGE than those on a lower dose. Hence, thiazolidinedione is more effective in raising esRAGE and total sRAGE level than sulfonylurea, and treatment modality contributes to modulation of esRAGE level to a greater extent than improvement in glycaemic control. Taken together, our data suggest that thiazolidinedione may have a direct effect on esRAGE and sRAGE.

The mechanism(s) whereby rosiglitazone increases serum esRAGE and total sRAGE are unclear. Our data suggest that the effect of rosiglitazone on esRAGE and sRAGE is unlikely to be mediated by adiponectin, as there was no correlation between the changes in esRAGE or total sRAGE and the increase in adiponectin. Thiazolidinediones activate the transcription factor PPAR $\gamma$; it has, moreover, been shown that thiazolidinedione can reduce $R A G E$ mRNA and protein expression in endothelial cells, as well as in aortic smooth muscle cells in vitro [16, 17]. However, the effect of thiazolidinedione on sRAGE or esRAGE production has not been examined and it remains to be determined whether thiazolidinedione can alter the pattern 
of production of the different RAGE isoforms such that production of full-length RAGE is decreased and production of sRAGE is increased. Forbes et al. have provided evidence that $R A G E$ expression can be modulated by showing that, in diabetic rats treated with ACE inhibitor, there was a specific increase in sRAGE production, which was associated with a decrease in full-length RAGE in renal tissues [22]. They also showed that inhibition of the reninangiotensin system by ACE inhibitors in patients with type 1 diabetes increased plasma sRAGE levels.

RAGE is a multi-ligand receptor and RAGE ligands may potentially affect sRAGE and esRAGE levels. Other than AGE, RAGE also binds amyloidogenic peptides/polypeptides, amphoterins and S100/calgranulins. In this context, Basta et al. [15] have recently shown in a cross-sectional study that plasma S100A12 level is a determinant of plasma sRAGE concentration in type 2 diabetic patients. We only measured AGE in our study. The dominant AGE epitope for binding to RAGE is $N^{\varepsilon}$-(carboxymethyl)lysine (CML). The polyclonal anti-AGE antibody raised against AGERNase that we used in our assay has been shown to recognise CML as well as other AGE epitopes, including non-fluorescent crosslink arginine-lysine imidazole [23, 24]. Hence, our assay was able to detect most of the circulating AGE. We did not find a significant relationship between the reduction in AGE and the changes in sRAGE and esRAGE. Moreover, the contribution of improved glycaemic control to the raising of sRAGE levels was relatively minor compared with the effect of thiazolidinedione. Interestingly, however, there was an association of the changes in MDA with sRAGE and esRAGE in the sulfonylurea-treated patients, suggesting that oxidative stress may potentially affect sRAGE levels.

This is the first study to measure both total sRAGE and esRAGE in the same group of diabetic individuals. The rationale for doing so was that there are distinct sRAGE species and it has been suggested that esRAGE might constitute only part, but not all of human sRAGE in serum. We have shown that the serum concentration of esRAGE is approximately onethird of the level of serum total sRAGE and the levels of esRAGE and sRAGE are correlated. With the assays currently available, it is difficult to delineate the specific cellular source(s) of sRAGE species. It is not clear whether measuring a distinct splice variant of sRAGE, such as esRAGE, would be a more useful marker or predictor of diabetic vascular complications than measuring total sRAGE. Antibodies and corresponding assays for the detection of discrete splice variants of sRAGE need to be developed before the specific role of sRAGE species as a biomarker of diabetic complications and their respective roles in the pathogenesis of diabetic complications can be fully determined. The effectiveness of upregulating endogenous sRAGE species to protect tissues against the harmful effects of AGE also needs to be investigated.
In conclusion, we have shown that treating type 2 diabetic patients with thiazolidinedione can increase circulating esRAGE and total sRAGE levels. Whether modulating circulating sRAGE can prevent or delay the progression of diabetic complications will have to be evaluated in longterm prospective studies.

Acknowledgements This study was supported by funding from the Hong Kong Research Grants Council to K. Tan (HKU7350/02) and K. Lam (HKU7637/05M). The authors are grateful to G. Cheung and C. Fong for their assistance in the clinical studies, and to S. Shiu for his technical assistance.

Duality of interest The authors declare that there is no duality of interest associated with this manuscript.

\section{References}

1. Schmidt AM, Yan SD, Wautier JL, Stern D (1999) Activation of receptor for advanced glycation end products: a mechanism for chronic vascular dysfunction in diabetic vasculopathy and atherosclerosis. Circ Res 84:489-497

2. Singh R, Barden A, Mori T, Beilin L (2001) Advanced glycation end-products: a review. Diabetologia 44:129-146

3. Bucciarelli LG, Wendt T, Rong L et al (2002) RAGE is a multiligand receptor of the immunoglobulin superfamily: implications for homeostasis and chronic disease. Cell Mol Life Sci 59:1117-1128

4. Yonekura H, Yamamoto Y, Sakurai S et al (2003) Novel splice variants of the receptor for advanced glycation end-products expressed in human vascular endothelial cells and pericytes, and their putative roles in diabetes-induced vascular injury. Biochem $\mathrm{J}$ 370:1097-1109

5. Schlueter C, Hauke S, Flohr AM, Rogalla P, Bullerdiek J (2003) Tissue-specific expression patterns of the RAGE receptor and its soluble forms - a result of regulated alternative splicing? Biochim Biophys Acta 1630:1-6

6. Park IH, Yeon SI, Youn JH et al (2004) Expression of a novel secreted splice variant of the receptor for advanced glycation end products (RAGE) in human brain astrocytes and peripheral blood mononuclear cells. Mol Immunol 40:1203-1211

7. Yamamoto Y, Yamagishi S, Yonekura H et al (2000) Roles of the AGE-RAGE system in vascular injury in diabetes. Ann N Y Acad Sci 902:163-170

8. Wautier JL, Zoukourian C, Chappey O et al (1996) Receptormediated endothelial cell dysfunction in diabetic vasculopathy. Soluble receptor for advanced glycation end products blocks hyperpermeability in diabetic rats. J Clin Invest 97:238-243

9. Park L, Raman KG, Lee KJ et al (1998) Suppression of accelerated diabetic atherosclerosis by the soluble receptor for advanced glycation endproducts. Nat Med 4:1025-1031

10. Goova MT, Li J, Kislinger T et al (2001) Blockade of receptor for advanced glycation end-products restores effective wound healing in diabetic mice. Am J Pathol 159:513-525

11. Challier M, Jacqueminet S, Benabdesselam O, Grimaldi A, Beaudeux JL (2005) Increased serum concentrations of soluble receptor for advanced glycation endproducts in patients with type 1 diabetes. Clin Chem 51:1749-1750

12. Tan KC, Shiu SW, Chow WS, Leng L, Bucala R, Betteridge DJ (2006) Association between serum level of soluble receptor for advanced glycation end products and circulating advanced glycation end products in type 2 diabetes. Diabetologia 49:2756-2762 
13. Katakami N, Matsuhisa M, Kaneto H et al (2005) Decreased endogenous secretory advanced glycation end product receptor in type 1 diabetic patients: its possible association with diabetic vascular complications. Diabetes Care 28:2716-2721

14. Koyama H, Shoji T, Yokoyama H et al (2005) Plasma level of endogenous secretory RAGE is associated with components of the metabolic syndrome and atherosclerosis. Arterioscler Thromb Vasc Biol 25:2587-2593

15. Basta G, Sironi AM, Lazzerini G et al (2006) Circulating soluble receptor for advanced glycation end products is inversely associated with glycemic control and S100A12 protein. J Clin Endocrinol Metab 91:4528-4634

16. Marx N, Walcher D, Ivanova N et al (2004) Thiazolidinediones reduce endothelial expression of receptors for advanced glycation end products. Diabetes 53:2662-2668

17. Wang K, Zhou Z, Zhang M et al (2006) Peroxisome proliferatoractivated receptor gamma down-regulates receptor for advanced glycation end products and inhibits smooth muscle cell proliferation in a diabetic and nondiabetic rat carotid artery injury model. J Pharmacol Exp Ther 317:37-43

18. Tan KCB, Chow WS, Ai VHG, Metz C, Bucala R, Lam KSL (2002) Advanced glycation end products and endothelial dysfunction in type 2 diabetes mellitus. Diabetes Care 25:1055-1059
19. Tan KCB, Xu A, Chow WS et al (2004) Hypoadiponectinemia is associated with impaired endothelial vasomotor function. J Clin Endocrinol Metab 89:765-769

20. Hanford LE, Enghild JJ, Valnickova Z et al (2004) Purification and characterization of mouse soluble receptor for advanced glycation end products (sRAGE). J Biol Chem 279:5001950024

21. Hudson BI, Harja E, Moser B, Schmidt AM (2005) Soluble levels of receptor for advanced glycation endproducts (sRAGE) and coronary artery disease: the next C-reactive protein? Arterioscler Thromb Vasc Biol 25:879-882

22. Forbes JM, Thorpe SR, Thallas-Bonke V et al (2005) Modulation of soluble receptor for advanced glycation end products by angiotensin-converting enzyme-1 inhibition in diabetic nephropathy. J Am Soc Nephrol 16:2363-2372

23. Berg TJ, Clausen JT, Torjesen PA et al (1998) The advanced glycation end product Nepsilon-(carboxymethyl)lysine is increased in serum from children and adolescents with type 1 diabetes. Diabetes Care 21:1997-2002

24. Al-Abed Y, Bucala R (2000) Structure of a synthetic glucose derived advanced glycation end product that is immunologically cross-reactive with its naturally occurring counterparts. Bioconjugate Chem 11:39-45 